\title{
Produção da bananeira 'Nanicão' em diferentes densidades de plantas e sistemas de espaçamento ${ }^{(1)}$
}

\begin{abstract}
João Alexio Scarpare Filho( ${ }^{(2)}$ e Ricardo Alfredo Kluge ${ }^{(2)}$
Resumo - O objetivo do presente trabalho foi verificar o efeito de diferentes densidades de plantio e sistemas de espaçamento sobre a produção da bananeira 'Nanicão', avaliando-se os primeiros quatro ciclos. Quatro densidades (3.333, 2.222, 1.666 e 1.333 plantas ha $^{-1}$ ) e dois sistemas de espaçamento (retângulo e triângulo) foram testados para as condições de Piracicaba, SP. O aumento da densidade de 1.333 para 3.333 plantas ha ${ }^{-1}$ diminuiu a massa do cacho em $15 \%$ a $20 \%$, em decorrência do menor número de frutos por cacho, massa e tamanho do fruto. A produção foi sempre maior com o aumento da densidade, porém não ocorreu o mesmo quanto à produtividade. Até o terceiro ciclo, a produtividade das plantas no tratamento de maior densidade superou a das demais. No quarto ciclo, não houve diferença de produtividade entre as plantas, nas densidades testadas, em razão do aumento na duração do ciclo de produção do plantio mais denso. Comparado com o retângulo, o sistema de espaçamento em triângulo promoveu maior massa do cacho no primeiro ciclo e produtividade levemente superior ao longo dos quatro ciclos.
\end{abstract}

Termos para indexação: Musa, espaçamento, produção, frutas, dimensão, peso.

\section{Yield of 'nanicão' banana at different plant densities and spacing systems}

\begin{abstract}
The objective of this research was to study the effect of different planting densities and spacing systems on the yield of the 'Nanicão' banana, evaluating the first four cycles. Four density $\left(3,333 ; 2,222 ; 1,666\right.$ and 1,333 plants ha $\left.^{-1}\right)$ and two spacing systems (rectangle and triangle) were tested for the conditions of Piracicaba, SP, Brazil. The increase of the density of 1,333 to 3,333 plants ha- ${ }^{-1}$ decreased bunch weight in $15 \%$ to $20 \%$, due to reduction in number of fruit by bunch and fruit size. The yield was always higher as the density increased; however, there was not the same behavior for the productivity. Until the third cycle, the highest plant density overcame the productivity of the others. In the fourth cycle there was no difference in productivity among the densities, due to the increase in the duration of crop cycle in the highest density. The spacing system in triangle promoted higher bunch weight in the first cycle and productivity slightly higher along the cycles if compared with rectangle system.
\end{abstract}

Index terms: Musa, spacing, production, fruits, dimension, weight.

\section{Introdução}

A bananicultura encontra-se amplamente difundida pelas diversas regiões do Brasil, sendo encontrada desde as regiões mais quentes, como o Norte e o Nordeste, até as regiões mais frias, como o litoral do Rio Grande do Sul e Santa Catarina (Simão, 1998).

\footnotetext{
(1) Aceito para publicação em 7 de fevereiro de 2000.

(2) Escola Superior de Agricultura Luiz de Queiros, Dep. de Ciências Biológicas, Caixa Postal 9, CEP 13418-900 Piracicaba, SP. E-mail: jascarpa@carpa.ciagri.usp.br, rakluge@ carpa.ciagri.usp.br
}

No Estado de São Paulo, a bananicultura do planalto vem ganhando força, aliando-se ao grande volume de produção de bananas provenientes do Vale do Ribeira, no litoral.

O espaçamento ideal de plantio para a bananeira é dependente de vários fatores, como porte da cultivar, fertilidade do solo, seleção de seguidores (rebentos), nível de tecnologia aplicada, controle de plantas daninhas, velocidade do vento, topografia, condições de mercado, longevidade do bananal, destino das frutas e outros (Simmonds, 1982; Lichtemberg, 1984; Stover \& Simmonds, 1987; Soto Ballestero et al., 1992). Além disto, a resposta das plantas às diferentes populações depende das condi- 
ções climáticas da região (Stover \& Simmonds 1987; Robinson \& Nel, 1988; Simão, 1998). Todo este complexo de fatores tem influência na escolha das densidades a serem utilizadas para as bananeiras, apresentando uma amplitude de 375 a 5.000 plantas ha- $^{-1}$ nas diversas regiões do mundo.

Robinson (1995) comenta que para as regiões mais quentes, de clima tropical, as altas densidades (mais de 3.000 plantas ha $^{-1}$ ) podem ser utilizadas para maximizar a produção e fornecer proteção contra temperaturas demasiadamente altas. Em regiões de clima subtropical, com invernos frios, as baixas densidades (menos de 2.000 plantas ha-1 $^{-1}$ ) são as preferidas, como forma de aproveitar melhor a disponibilidade térmica para o crescimento e evitar o aumento no ciclo.

O adensamento de plantio de plantas frutíferas tem sido visto como uma tendência mundial, pois promove melhor aproveitamento do solo, mão-de-obra e insumos, e aumento na produção por área. Para sua utilização é necessário estabelecê-lo em regiões de clima favorável à cultura e viabilizar a instalação de sistema de irrigação, considerando que o adensamento promove maior competição interplanta por fatores de crescimento (água, luz e nutrientes). Além disso, para a adoção de plantios em altas densidades, é necessário analisar os impactos que uma maior população de plantas pode trazer sobre a produtividade e os componentes de produção.

Diversas pesquisas têm sido relatadas mostrando o efeito do uso de diferentes densidades de plantio sobre os componentes de produção e a produtividade da bananeira. Os resultados encontrados são variados, de acordo com a cultivar, clima da região, espaçamentos e densidades, e número de ciclos avaliados.

Tem sido constatado que a duração do ciclo é maior com o aumento da densidade de plantas, principalmente após a primeira safra (Melin et al., 1976; Chundawat et al., 1983; Gomes et al., 1984; Chattopadhyay et al., 1985; Daniells et al., 1985; Lichtemberg et al., 1990, 1998). Este maior intervalo para o ciclo da bananeira deve-se, segundo Robinson \& Nel (1986) e Israeli et al. (1995), ao maior sombreamento no interior do bananal, o que provoca atraso e desuniformidade no desenvolvimento dos rebentos, e retarda a emissão da inflorescência.
Tem sido verificado que o aumento da densidade eleva a produção por hectare, em razão do grande número de cachos colhidos (Gomes et al., 1984; Daniells et al., 1985; Robinson \& Nel, 1986; Lichtemberg et al., 1988, 1997). Entretanto, considerando que a bananeira não apresenta ciclo anual definido, a produtividade, medida em $\mathrm{t} \mathrm{ha}^{-1} \mathrm{ano}^{-1}$, é progressivamente reduzida com o incremento da densidade e com a evolução dos ciclos (Daniells et al., 1985; Robinson \& Nel, 1988, 1989; Robinson, 1995; Lichtemberg et al., 1998).

Os componentes de produção (cachos, pencas e frutos) também sofrem influência da densidade de plantas. Normalmente, o aumento da densidade reduz a massa do cacho, principalmente após o primeiro ciclo (Mattos et al., 1970; Santos, 1977; Obiefuna et al., 1982; Chundawat et al., 1983; Gomes et al., 1984; Chattopadhyay et al., 1985; Daniells et al., 1985; Robinson \& Nel, 1986; Lichtemberg et al., 1998). Similarmente, o número de pencas e frutos por cacho é menor nas maiores densidades (Mattos et al., 1970; Obiefuna et al., 1982; Robinson \& Nel, 1989; Lichtemberg et al., 1990).

Além da densidade, a distribuição das plantas na área de plantio pode influenciar a produção, pois os diferentes sistemas de espaçamento modificam a incidência de luminosidade no interior da plantação (Janick, 1968; Pereira, 1989; Israeli et al., 1995). Segundo Robinson (1995), diferentes populações e distribuições das plantas na área de plantio afetam a eficiência fisiológica da planta, sobretudo sua capacidade fotossintética.

Diversos são os sistemas de espaçamento que podem ser utilizados para a bananeira, incluindo: quadrado, retângulo, triângulo eqüilátero, hexagonal, fileiras duplas, entre outros (Daniells et al., 1985; Robinson et al., 1989; Soto Ballestero et al., 1992). A opção por um desses sistemas depende do porte da cultivar, região, topografia e manejo do bananal (Robinson, 1995). Os sistemas em quadrado e retângulo, muito utilizados na bananicultura, são pouco eficientes quanto ao aproveitamento da luminosidade e do terreno, além de não serem aconselhados para declividades superiores a 4\% (Belalcázar Carvajal, 1991). Ao contrário, os demais sistemas apresentam maior eficiência de aproveitamento dos fatores de crescimento (luz, água e nutrientes), o que favorece 
a produção. Soto Ballestero et al. (1992) apontam que a distribuição em triângulo é a que promove o melhor aproveitamento desses fatores, porém sua utilização tem sido pouco difundida e seus efeitos sobre a produção de bananas necessitam ser melhor esclarecidos.

Com o aumento progressivo das áreas de plantio no planalto paulista, existe a necessidade de maiores conhecimentos sobre os efeitos de diferentes densidades e sistemas de espaçamento sobre a produção de bananas nesta região.

O objetivo do presente trabalho foi verificar, nas condições de Piracicaba, SP, o comportamento de bananeiras 'Nanicão' submetidas a quatro densidades de plantio e dois sistemas de espaçamento.

\section{MATERIAL E MÉTODOS}

O experimento foi conduzido no bananal experimental do Departamento de Produção Vegetal da Escola Superior de Agricultura "Luiz de Queiroz", situado no Município de Piracicaba, SP, Brasil.

O clima de Piracicaba, conforme a classificação de Köppen, é do tipo Cwa: tropical úmido, com três meses mais secos (junho, julho e agosto), chuvas de verão e seca no inverno. A temperatura média do mês mais quente é maior do que $22^{\circ} \mathrm{C}$ e do mês mais frio não é inferior a $16^{\circ} \mathrm{C}$, com média de $21,1^{\circ} \mathrm{C}$; precipitação média de $1.253 \mathrm{~mm} / \mathrm{ano}$; ventos predominantes 1 a este e 2 a sudoeste, com velocidade média de $2,2 \mathrm{~m} / \mathrm{s}$; umidade relativa do ar de $74 \%$ e insolação mensal média de 201,5 horas (2.418 lux ano $\left.{ }^{-1}\right)$. A altitude de Piracicaba é de $546 \mathrm{~m}$.

$\mathrm{O}$ solo da área experimental é classificado como Terra Roxa Estruturada, eutrófica, A moderado, textura argilosa (Kandudalfic Eutrudox), segundo Vidal-Torrado \& Sparovek (1993).

Foram estudados os primeiros quatro ciclos da cultura da bananeira 'Nanicão' (Musa AAA subgrupo Cavendish), estabelecida em 24 de janeiro de 1994. A cultivar, uma mutação da 'Nanica', apresenta porte mais alto, atingindo de 2,20 a 3,20 m. Foram utilizadas mudas tipo "chifrão" (brotação lateral, com folhas lanceoladas e emitindo folhas normais, com massa média de $2,5 \mathrm{~kg}$ ). O bananal foi conduzido com três plantas por touceira (planta "mãe", "filha" e "neta").

Durante os ciclos da cultura foram realizadas as práticas de controle de plantas daninhas, capinas, desbaste de rebentos, retirada de folhas velhas, escoramento da planta, retirada do coração, controle de pragas, doenças, e adubações. As adubações consistiram na aplicação de
$2.000 \mathrm{~kg} \mathrm{ha}^{-1} \mathrm{ano}^{-1}$ de N, P e K na formulação 14-7-28, parcelada em quatro vezes, sendo realizadas nos meses de setembro, novembro, janeiro e março.

Os tratamentos consistiram do uso de diferentes densidades e sistemas de espaçamento. Os sistemas de espaçamento utilizados foram retângulo e triângulo (quincôncio). Cada arranjo de plantas apresentou quatro densidades de plantio: 1.333, 1.666, 2.222 e 3.333 plantas ha ${ }^{-1}$, representando um total de oito tratamentos. No arranjo em retângulo, as dimensões utilizadas foram $3,0 \mathrm{~m} \times 2,5 \mathrm{~m}, 3,0 \mathrm{~m} \times 2,0 \mathrm{~m}, 3,0 \mathrm{~m} \times 1,5 \mathrm{~m}$ e $3,0 \mathrm{~m} \times$ $1,0 \mathrm{~m}$. No arranjo em triângulo, as dimensões (base $\mathrm{x}$ altura) foram: $2,8 \mathrm{~m} \times 1,4 \mathrm{~m}, 2,6 \mathrm{~m} \times 1,3 \mathrm{~m}, 2,4 \mathrm{~m} \times 1,2 \mathrm{me}$ $2,0 \mathrm{~m} \times 1,0 \mathrm{~m}$. Em cada vértice do retângulo e do triângulo foi colocada uma planta. $\mathrm{O}$ delineamento experimental adotado foi o de blocos casualizados, em esquema fatorial $4 \times 2$ (quatro densidades e dois sistemas de espaçamento), com três repetições. Cada parcela, incluindo a bordadura, foi constituída de 30 plantas, sendo consideradas 10 plantas úteis, o que totalizou 240 plantas avaliadas. Os dados coletados foram submetidos à análise de variância (teste $\mathrm{F}$ ) e, em caso de significância, as médias foram comparadas pelo teste de Tukey a $5 \%$ de probabilidade.

As seguintes variáveis foram analisadas: a) massa média do cacho $(\mathrm{kg})$ : cada cacho foi pesado logo após a colheita; b) número de pencas e frutos por cacho: por contagem; c) comprimento do fruto $(\mathrm{cm})$ : foi determinado com uma trena metálica flexível o comprimento de 10 frutos por cacho, da $2^{\mathrm{a}}$ penca superior, sendo medida a face convexa do fruto; d) massa média do fruto (g): foi calculado dividindo-se a massa do cacho (descontando a massa da ráquis) pelo número de frutos presentes no cacho; e) produção estimada por hectare $\left(\mathrm{t} \mathrm{ha}^{-1}\right)$ : em cada ciclo, a produção por hectare foi calculada multiplicando-se a massa do cacho pelo número de plantas por hectare.

Ao final do quarto ciclo, foi determinado o número de cachos produzidos por ano, a variação da massa do cacho em razão do mês de colheita, e a produtividade estimada, em $\mathrm{t} \mathrm{ha}^{-1} \mathrm{ano}^{-1}$. A produtividade foi determinada em cada ciclo, usando-se a fórmula preconizada por Robinson \& Nel (1988): $\mathrm{t} \mathrm{ha}^{-1} \mathrm{ano}^{-1}=\left[\right.$ produção $\left(\mathrm{t} \mathrm{ha}^{-1}\right) /$ duração do ciclo (meses)] x 12.

\section{Resultados e Discussão}

$\mathrm{O}$ aumento da densidade reduziu significativamente a massa do cacho a partir do primeiro ciclo (Tabela 1). No geral, a massa do cacho observada na densidade de 3.333 plantas ha ${ }^{-1}$ foi $15 \%$ a $20 \%$ inferior à observada nas densidades de 1.666 e 1.333 plan- 
tas ha-1 ${ }^{-1}$ A massa do cacho observada com 2.222 plantas ha ${ }^{-1}$ teve comportamento intermediário, às vezes diferindo e às vezes não diferindo do plantio mais denso. A distribuição em triângulo favoreceu a massa do cacho apenas no primeiro ciclo. Os cachos colhidos neste sistema apresentaram massa superior, em torno de $10 \%$, em relação aos colhidos no retângulo. Observou-se, também, que a massa do cacho aumentou no terceiro ciclo; este resultado foi decorrente da época da colheita. A maioria dos cachos desse ciclo foi colhida no outono e inverno. Segundo Kluge (1999), na região de Piracicaba, essas estações favorecem a obtenção de maior número de frutos e maior massa do cacho, como decorrência de condições mais favoráveis de temperatura e disponibilidade hídrica que ocorrem durante o período vegetativo e iniciação floral.

O número de pencas observado no experimento foi, em média, de nove por cacho, na densidade de

Tabela 1. Massa média ( $\mathrm{kg}$ ) do cacho de bananeiras 'Nanicão' submetidas a diferentes densidades e sistemas de espaçamento, em Piracicaba, $\mathbf{S P}^{(1)}$.

\begin{tabular}{|c|c|c|c|c|c|}
\hline \multirow{2}{*}{$\begin{array}{l}\text { Sistema de } \\
\text { espaçamento }\end{array}$} & \multicolumn{4}{|c|}{ Número de plantas ha $^{-1}$} & \multirow[t]{2}{*}{ Média } \\
\hline & 3.333 & 2.222 & 1.666 & 1.333 & \\
\hline \multicolumn{6}{|c|}{$1^{\circ}$ ciclo } \\
\hline Retângulo & 22,87 & 24,01 & 26,47 & 26,69 & $25,01 b$ \\
\hline Triângulo & 24,93 & 27,90 & 28,00 & 29,97 & $27,70 \mathrm{a}$ \\
\hline Média & $23,90 \mathrm{C}$ & $25,95 \mathrm{~B}$ & $27,23 \mathrm{AB}$ & $28,33 \mathrm{~A}$ & \\
\hline \multicolumn{6}{|c|}{$2^{-}$ciclo } \\
\hline Retângulo & 22,50 & 23,08 & 23,95 & 25,52 & $23,76 a$ \\
\hline Triângulo & 21,91 & 23,79 & 22,93 & 26,14 & $23,69 \mathrm{a}$ \\
\hline Média & $22,20 \mathrm{~B}$ & $23,43 \mathrm{AB}$ & $23,44 \mathrm{AB}$ & $25,83 \mathrm{~A}$ & \\
\hline \multicolumn{6}{|c|}{$3^{\circ}$ ciclo } \\
\hline Retângulo & 23,42 & 28,73 & 30,72 & 29,81 & $28,17 \mathrm{a}$ \\
\hline Triângulo & 26,16 & 29,47 & 28,95 & 30,78 & $28,84 \mathrm{a}$ \\
\hline Média & $24,79 \mathrm{~B}$ & $29,10 \mathrm{~A}$ & $29,83 \mathrm{~A}$ & $30,29 \mathrm{~A}$ & \\
\hline \multicolumn{6}{|c|}{$4^{\circ}$ ciclo } \\
\hline Retângulo & 17,87 & 24,30 & 24,27 & 29,60 & $24,01 \mathrm{a}$ \\
\hline Triângulo & 19,04 & 24,25 & 27,02 & 29,17 & $24,87 \mathrm{a}$ \\
\hline Média & $18,45 \mathrm{C}$ & $24,28 B$ & $25,64 \mathrm{~B}$ & $29,38 \mathrm{~A}$ & \\
\hline
\end{tabular}

3.333 plantas ha-1, e de dez pencas por cacho, nas demais densidades; não houve efeito do sistema de espaçamento.

Em relação ao número de frutos por cacho, observou-se uma tendência de haver mais frutos nos cachos colhidos nas menores densidades, principalmente na densidade de 1.333 plantas ha ${ }^{-1}$, onde o número de frutos foi maior que nas demais, embora não tenha diferido estatisticamente da densidade de 1.666 plantas ha-1, exceto no quarto ciclo (Tabela 2).

A massa e o comprimento médio do fruto foram altamente influenciados pela densidade das plantas (Tabelas 3 e 4), e os frutos obtidos com 3.333 plantas ha ${ }^{-1}$ apresentaram os menores valores para essas duas características. O sistema de espaçamento interferiu significativamente na massa média apenas no primeiro ciclo, onde os frutos colhidos no triângulo apresentaram massa superior à obtida no retângulo, em, aproximadamente, $8 \%$.

Tabela 2. Número de frutos por cacho de bananeiras 'Nanicão' submetidas a diferentes densidades e sistemas de espaçamento, em Piracicaba, $\mathrm{SP}^{(1)}$.

\begin{tabular}{|c|c|c|c|c|c|}
\hline \multirow{2}{*}{$\begin{array}{l}\text { Sistema de } \\
\text { espaçamento }\end{array}$} & \multicolumn{4}{|c|}{ Número de plantas ha ${ }^{-1}$} & \multirow[t]{2}{*}{ Média } \\
\hline & 3.333 & 2.222 & 1.666 & 1.333 & \\
\hline \multicolumn{6}{|c|}{$1^{\circ}$ ciclo } \\
\hline Retângulo & 118 & 117 & 125 & 128 & $122 \mathrm{a}$ \\
\hline Triângulo & 119 & 127 & 123 & 133 & $126 \mathrm{a}$ \\
\hline Média & 119B & $122 \mathrm{AB}$ & $124 \mathrm{AB}$ & $130 \mathrm{~A}$ & \\
\hline \multicolumn{6}{|c|}{$2^{\underline{0} \text { ciclo }}$} \\
\hline Retângulo & 141 & 137 & 136 & 144 & $140 \mathrm{a}$ \\
\hline Triângulo & 135 & 137 & 131 & 146 & $137 \mathrm{a}$ \\
\hline Média & $138 \mathrm{~A}$ & $137 \mathrm{~A}$ & $133 \mathrm{~A}$ & $145 \mathrm{~A}$ & \\
\hline \multicolumn{6}{|c|}{$3^{-0}$ ciclo } \\
\hline Retângulo & 166 & 183 & 181 & 189 & $180 \mathrm{a}$ \\
\hline Triângulo & 168 & 190 & 179 & 188 & $181 \mathrm{a}$ \\
\hline Média & 167B & $186 \mathrm{~A}$ & $180 \mathrm{AB}$ & $189 \mathrm{~A}$ & \\
\hline \multicolumn{6}{|c|}{$4^{\circ}$ ciclo } \\
\hline Retângulo & 130 & 159 & 155 & 180 & $156 a$ \\
\hline Triângulo & 150 & 164 & 163 & 180 & $164 a$ \\
\hline Média & $140 \mathrm{C}$ & $162 \mathrm{AB}$ & 159BC & $180 \mathrm{~A}$ & \\
\hline
\end{tabular}


Os resultados obtidos quanto ao número de pencas e frutos por cacho, massa e comprimento médio do fruto causaram as diferenças verificadas na massa do cacho. Assim, a menor massa de cacho verificada na maior densidade pode ser creditada ao menor número de pencas e frutos, bem como à menor massa $\mathrm{e}$ tamanho do fruto. O comportamento verificado na menor densidade é o oposto. A diminuição da massa do cacho com o aumento da densidade de plantas também foi verificada por outros autores (Mattos et al., 1970; Santos, 1977; Obiefuna et al., 1982; Daniells et al., 1985; Lichtemberg et al., 1988, 1990; Robinson \& Nel, 1988).

O adensamento normalmente provoca incremento na altura das plantas em razão da maior competição por luz (Kholi et al., 1976; Melin et al., 1976; Chattopadhyay et al., 1985; Lichtemberg et al., 1988, 1997). Isto promove, provavelmente, uma menor alocação de água, nutrientes e reguladores vegetais aos componentes de produção, para compensar o maior crescimento do pseudocaule. Como resultado,

Tabela 3. Massa média (g) do fruto de bananeiras 'Nanicão' submetidas a diferentes densidades e sistemas de espaçamento, em Piracicaba, $\mathrm{SP}^{(1)}$.

\begin{tabular}{|c|c|c|c|c|c|}
\hline \multirow{2}{*}{$\begin{array}{l}\text { Sistema de } \\
\text { espaçamento }\end{array}$} & \multicolumn{4}{|c|}{ Número de plantas ha ${ }^{-1}$} & \multirow[t]{2}{*}{ Média } \\
\hline & 3.333 & 2.222 & 1.666 & 1.333 & \\
\hline & \multicolumn{5}{|c|}{$1^{\circ}$ ciclo } \\
\hline Retângulo & 177,5 & 187,9 & 193,8 & 191,8 & $187,8 b$ \\
\hline Triângulo & 191,5 & 203,0 & 211,8 & 206,2 & $203,1 \mathrm{a}$ \\
\hline \multirow[t]{2}{*}{ Média } & $184,5 \mathrm{~B}$ & $195,5 \mathrm{AB}$ & $202,8 \mathrm{~A}$ & $199,0 \mathrm{~A}$ & \\
\hline & & \multicolumn{4}{|c|}{$2^{\circ}$ ciclo } \\
\hline Retângulo & 146,9 & 156,7 & 162,0 & 162,7 & $157,0 \mathrm{a}$ \\
\hline Triângulo & 150,8 & 158,7 & 161,9 & 163,2 & $158,4 \mathrm{a}$ \\
\hline \multirow[t]{2}{*}{ Média } & $148,9 \mathrm{~B}$ & $157,7 \mathrm{AB}$ & $161,5 \mathrm{AB}$ & $163,0 \mathrm{~A}$ & \\
\hline & & \multicolumn{4}{|c|}{$3^{\circ}$ ciclo } \\
\hline Retângulo & 129,87 & 143,66 & 156,24 & 144,65 & $143,60 \mathrm{a}$ \\
\hline Triângulo & 144,28 & 142,63 & 148,43 & 151,18 & $146,63 \mathrm{a}$ \\
\hline \multirow[t]{2}{*}{ Média } & $137,07 \mathrm{~B}$ & $143,14 \mathrm{AB}$ & $152,33 \mathrm{~A}$ & $147,91 \mathrm{~A}$ & \\
\hline & & \multicolumn{4}{|c|}{$4^{\mathrm{o}}$ ciclo } \\
\hline Retângulo & 125,4 & 140,6 & 144,0 & 151,3 & $140,3 \mathrm{a}$ \\
\hline Triângulo & 116,8 & 136,0 & 152,5 & 149,1 & $138,6 \mathrm{a}$ \\
\hline Média & $121,1 \mathrm{C}$ & $138,3 \mathrm{~B}$ & $148,3 \mathrm{AB}$ & $150,2 \mathrm{~A}$ & \\
\hline
\end{tabular}

menor quantidade de frutos é produzida, e sua dimensão é menor, resultando num cacho de menor massa. Janick (1968) comentou que as partes economicamente importantes das plantas (frutos, sementes, tubérculos, etc) podem reagir às pressões de uma população crescente, após iniciada a competição, pela diminuição do seu tamanho, do seu número, ou de ambos.

A diminuição da massa do cacho nas altas densidades também pode ser atribuída à redução da capacidade fotossintética da planta como resposta ao sombreamento, que reduz o enchimento e o desenvolvimento dos frutos (Robinson \& Nel, 1989). Israeli et al. (1995) relataram que o sombreamento de bananeiras 'Grand Nain' pode reduzir em até $50 \%$ a massa do cacho, a depender do nível de sombreamento. Turner (1994) comentou que os efeitos da alteração da densidade de bananeiras sobre a massa do cacho é bastante complexa, apontando que o aumento da densidade reduz a massa do cacho por afetar a temperatura ótima para o crescimento que,

Tabela 4. Comprimento médio $(\mathrm{cm})$ do fruto de bananeiras 'Nanicão' submetidas a diferentes densidades e sistemas de espaçamento, em Piracicaba, $\mathrm{SP}^{(1)}$.

\begin{tabular}{|c|c|c|c|c|c|}
\hline \multirow{2}{*}{$\begin{array}{l}\text { Sistema de } \\
\text { espaçamento }\end{array}$} & \multicolumn{4}{|c|}{ Número de plantas ha ${ }^{-1}$} & \multirow[t]{2}{*}{ Média } \\
\hline & 3.333 & 2.222 & 1.666 & 1.333 & \\
\hline & \multicolumn{5}{|c|}{$1^{\circ}$ ciclo } \\
\hline Retângulo & 20,9 & 23,1 & 23,4 & 23,3 & $22,7 \mathrm{a}$ \\
\hline Triângulo & 20,3 & 24,0 & 24,4 & 24,1 & $23,2 \mathrm{a}$ \\
\hline \multirow[t]{2}{*}{ Média } & $20,6 \mathrm{~B}$ & $23,5 \mathrm{~A}$ & $23,9 \mathrm{~A}$ & $23,7 \mathrm{~A}$ & \\
\hline & \multicolumn{5}{|c|}{$2^{\circ}$ ciclo } \\
\hline Retângulo & 19,5 & 21,2 & 21,4 & 21,5 & $20,9 a$ \\
\hline Triângulo & 19,8 & 21,3 & 21,6 & 21,7 & $21,1 \mathrm{a}$ \\
\hline \multirow[t]{2}{*}{ Média } & $19,7 \mathrm{~B}$ & $21,3 \mathrm{~A}$ & $21,5 \mathrm{~A}$ & $21,6 \mathrm{~A}$ & \\
\hline & \multicolumn{5}{|c|}{$3^{\circ}$ ciclo } \\
\hline Retângulo & 19,0 & 19,8 & 21,0 & 20,8 & $20,2 \mathrm{a}$ \\
\hline Triângulo & 18,9 & 19,4 & 20,8 & 21,4 & $20,1 \mathrm{a}$ \\
\hline \multirow[t]{2}{*}{ Média } & $19,0 \mathrm{~B}$ & $19,6 \mathrm{~B}$ & $20,9 \mathrm{~A}$ & $21,1 \mathrm{~A}$ & \\
\hline & \multicolumn{5}{|c|}{$4^{\circ}$ ciclo } \\
\hline Retângulo & 18,6 & 19,9 & 20,2 & 21,6 & $20,1 \mathrm{a}$ \\
\hline Triângulo & 19,0 & 20,1 & 20,7 & 21,7 & $20,4 \mathrm{a}$ \\
\hline Média & $18,8 \mathrm{C}$ & $20,0 \mathrm{BC}$ & $20,5 \mathrm{AB}$ & $21,7 \mathrm{~A}$ & \\
\hline
\end{tabular}

Pesq. agropec. bras., Brasília, v. 36, n. 1, p. 105-113, jan. 2001 
por sua vez, incide sobre a taxa de produção de folhas, número total de folhas produzidas e, possivelmente, taxa de emergência dos rebentos.

Nas densidades 1.333 e 1.666 plantas ha $^{-1}$ foi colhido um cacho por ano, na média dos quatro ciclos, enquanto nas duas maiores densidades $(2.222 \mathrm{e}$ 3.333 plantas $\mathrm{a}^{-1}$ ) não foi possível colher uma produção por ano (Tabela 5). Com base nesta observação, ficou claro que para calcular a produtividade por hectare dos bananais submetidos a diferentes densidades, não basta multiplicar a massa do cacho pelo número de plantas na área, determinando t $\mathrm{ha}^{-1}$. Essa determinação não é eficiente, pois o ciclo da bananeira não é anual e é pouco regular, principalmente em regiões onde há oscilações acentuadas de temperatura e pluviosidade. Para o cálculo de estimativa de produtividade, três componentes devem ser tomados: massa do cacho, população de plantas por hectare e duração do ciclo. Essa observação é corroborada por Robinson \& Nel (1988).

Ao calcular-se a produção por hectare, sem levar em consideração a duração do ciclo, verificou-se que as maiores densidades sempre superaram as menores em todos os ciclos avaliados (Tabela 6). Por outro lado, estimando a produtividade em $\mathrm{t} \mathrm{ha}^{-1} \mathrm{ano}^{-1}$, observou-se que os valores encontrados na densidade de 3.333 plantas ha ${ }^{-1}$ superaram os observados nas outras densidades até o terceiro ciclo, e igualaramse estatisticamente aos valores encontrados nas demais densidades do quarto ciclo (Tabela 7). Tal comportamento é decorrente do maior ciclo de produção verificado no plantio mais adensado (Tabela 8). O aumento na duração do ciclo é uma das respostas das plantas submetidas a altas populações, como consequiência da competição interplantas, a qual é inten-

Tabela 5. Cachos colhidos por ano em quatro ciclos de bananeiras 'Nanicão' submetidas a diferentes densidades e sistemas de espaçamento, em Piracicaba, $\mathrm{SP}^{(1)}$.

\begin{tabular}{|c|c|c|c|c|c|}
\hline \multirow{2}{*}{$\begin{array}{l}\text { Sistema de } \\
\text { espaçamento }\end{array}$} & \multicolumn{4}{|c|}{ Número de plantas ha ${ }^{-1}$} & \multirow[t]{2}{*}{ Média } \\
\hline & 3.333 & 2.222 & 1.666 & 1.333 & \\
\hline Retângulo & 0,86 & 0,95 & 1,01 & 1,05 & $0,97 \mathrm{a}$ \\
\hline Triângulo & 0,88 & 0,96 & 1,00 & 1,05 & $0,97 \mathrm{a}$ \\
\hline Média & $0,87 \mathrm{C}$ & $0,96 \mathrm{BC}$ & $1,01 \mathrm{AB}$ & $1,05 \mathrm{~A}$ & \\
\hline
\end{tabular}

sificada a partir da segunda safra (Gomes et al., 1984; Daniells et al., 1985; Robinson, 1995; Lichtemberg et al., 1996).

A distribuição de plantas no sistema de triângulo promoveu produtividade aproximadamente 5\% superior à do retângulo, na média dos ciclos estudados (Tabela 7). Comportamento que deve-se à pequena diferença observada quanto à massa do cacho, que revelou uma leve superioridade do triângulo sobre o retângulo.

A escolha da melhor densidade de plantio em relação a determinada cultivar de bananeira é complexa, e deve ser criteriosa, pois além dos fatores climáticos e tecnológicos envolvidos, deve-se também levar em consideração o manejo que será adotado, o mercado ao qual se destina a produção, e o próprio custo de produção.

Robinson (1995) considera que, se o bananal for explorado por poucos ciclos (até dois ou três ciclos), as altas densidades (mais de 3.000 plantas ha ${ }^{-1}$ ) podem ser utilizadas, pois há grande incremento na pro-

Tabela 6. Produção estimada $\left(\mathrm{t} \mathrm{ha}^{-1}\right)$ de bananeiras 'Nanicão' submetidas a diferentes densidades e sistemas de espaçamento, em Piracicaba, $\mathrm{SP}^{(1)}$.

\begin{tabular}{|c|c|c|c|c|c|}
\hline \multirow{2}{*}{$\begin{array}{l}\text { Sistema de } \\
\text { espaçamento }\end{array}$} & \multicolumn{4}{|c|}{ Número de plantas ha ${ }^{-1}$} & \multirow[t]{2}{*}{ Média } \\
\hline & 3.333 & 2.222 & 1.666 & 1.333 & \\
\hline & \multicolumn{5}{|c|}{$1^{\circ}$ ciclo } \\
\hline Retângulo & 76,22 & 53,35 & 44,11 & 35,58 & $52,32 \mathrm{~b}$ \\
\hline Triângulo & 83,06 & 62,00 & 46,64 & 39,96 & $57,92 \mathrm{a}$ \\
\hline \multirow[t]{2}{*}{ Média } & $79,65 \mathrm{~A}$ & $57,68 \mathrm{~B}$ & $45,38 \mathrm{C}$ & $37,77 \mathrm{C}$ & \\
\hline & \multicolumn{5}{|c|}{$2^{\circ}$ ciclo } \\
\hline Retângulo & 74,99 & 51,29 & 39,91 & 34,02 & $50,05 \mathrm{a}$ \\
\hline Triângulo & 73,02 & 52,85 & 38,21 & 34,84 & $49,73 \mathrm{a}$ \\
\hline \multirow[t]{2}{*}{ Média } & $74,01 \mathrm{~A}$ & $52,07 \mathrm{~B}$ & $39,06 \mathrm{C}$ & $34,43 \mathrm{C}$ & \\
\hline & \multicolumn{5}{|c|}{$3^{\circ}$ ciclo } \\
\hline Retângulo & 78,05 & 63,83 & 51,18 & 39,74 & $58,21 \mathrm{a}$ \\
\hline Triângulo & 87,19 & 65,48 & 48,23 & 41,03 & $60,50 \mathrm{a}$ \\
\hline \multirow[t]{2}{*}{ Média } & $82,62 \mathrm{~A}$ & $64,65 \mathrm{~B}$ & $49,70 \mathrm{C}$ & $40,38 \mathrm{C}$ & \\
\hline & \multicolumn{5}{|c|}{$4^{\circ}$ ciclo } \\
\hline Retângulo & 59,56 & 54,02 & 40,42 & 39,45 & $48,36 \mathrm{a}$ \\
\hline Triângulo & 63,46 & 53,89 & 45,02 & 38,89 & $50,31 \mathrm{a}$ \\
\hline Média & $61,51 \mathrm{~A}$ & $53,96 \mathrm{~A}$ & $42,72 \mathrm{~B}$ & $39,17 \mathrm{~B}$ & \\
\hline
\end{tabular}


dutividade. Ao contrário, na condução do bananal por vários ciclos, o autor não aconselha a adoção permanente do adensamento, pois com a evolução dos ciclos a produtividade sofre redução.

De acordo com o mercado, quando as frutas são destinadas à exportação ou a mercados mais exigentes em qualidade, o tamanho é um componente importante, e as frutas maiores apresentam maior valor comercial. Quando as frutas são destinadas aos mercados menos exigentes, é mais conveniente obter grande volume de produção e, neste caso, o adensamento pode ser utilizado nos primeiros ciclos, mesmo reduzindo o tamanho do fruto (Soto Ballestero et al., 1992).

Pelos resultados obtidos no presente trabalho, pode-se inferir que a adoção de alta densidade (3.333 plantas ha-1) é viável para a cultivar e região estudadas, desde que o destino da produção seja o mercado interno ou pouco exigente. Essa densidade apresenta, como vantagem, a excelente produtividade nos primeiros três ciclos, e como desvantagem, a

Tabela 7. Produtividade estimada $\left(\mathrm{t} \mathrm{ha}^{-1} \mathrm{ano}^{-1}\right)$ de bananeiras 'Nanicão' submetidas a diferentes densidades e sistemas de espaçamento, em Piracicaba, SP$^{(1)}$.

\begin{tabular}{|c|c|c|c|c|c|}
\hline \multirow{2}{*}{$\begin{array}{l}\text { Sistema de } \\
\text { espaçamento }\end{array}$} & \multicolumn{4}{|c|}{ Número de plantas $h^{-1}$} & \multirow[t]{2}{*}{ Média } \\
\hline & 3.333 & 2.222 & 1.666 & 1.333 & \\
\hline & \multicolumn{5}{|c|}{$1^{\circ}$ ciclo } \\
\hline Retângulo & 46,92 & 33,00 & 27,00 & 22,12 & $32,26 b$ \\
\hline Triângulo & 51,64 & 37,38 & 28,84 & 24,59 & $35,68 \mathrm{a}$ \\
\hline \multirow[t]{2}{*}{ Média } & $49,28 \mathrm{~A}$ & $35,19 \mathrm{BC}$ & $27,92 \mathrm{CD}$ & $23,35 \mathrm{D}$ & \\
\hline & \multicolumn{5}{|c|}{$2^{-}$ciclo } \\
\hline Retângulo & 84,89 & 64,78 & 53,81 & 53,71 & $64,29 \mathrm{a}$ \\
\hline Triângulo & 85,75 & 73,74 & 50,94 & 57,27 & $66,92 \mathrm{a}$ \\
\hline \multirow[t]{2}{*}{ Média } & $85,32 \mathrm{~A}$ & $69,26 \mathrm{~B}$ & $52,37 \mathrm{C}$ & $55,49 \mathrm{C}$ & \\
\hline & \multicolumn{5}{|c|}{$3^{\circ}$ ciclo } \\
\hline Retângulo & 72,60 & 66,03 & 61,41 & 48,66 & $62,17 \mathrm{a}$ \\
\hline Triângulo & 85,06 & 67,73 & 55,12 & 48,27 & $64,04 \mathrm{a}$ \\
\hline \multirow[t]{2}{*}{ Média } & $78,83 \mathrm{~A}$ & $66,88 \mathrm{~B}$ & $58,26 \mathrm{C}$ & $48,46 \mathrm{C}$ & \\
\hline & \multicolumn{5}{|c|}{$4^{\mathrm{o}}$ ciclo } \\
\hline Retângulo & 55,83 & 64,82 & 55,11 & 52,60 & $57,09 a$ \\
\hline Triângulo & 59,96 & 65,32 & 60,02 & 53,03 & $59,58 \mathrm{a}$ \\
\hline Média & $57,89 \mathrm{~A}$ & $65,07 \mathrm{~A}$ & $57,34 \mathrm{~A}$ & $52,81 \mathrm{~A}$ & \\
\hline
\end{tabular}

produção de frutos de menor tamanho. Pode-se manter esta população de plantas até o terceiro ciclo, e haverá a necessidade de reduzi-la, posteriormente, pela queda no rendimento anual, como resposta ao incremento do ciclo de produção. Nesse caso, podese reduzir o número de plantas de $1 / 3$ à metade após o terceiro ciclo, com base nos resultados obtidos no presente experimento para as densidades menores (2.222 e 1.666 plantas ha-1).

A adoção de baixas densidades (1.666 e 1.333 plantas ha $^{-1}$ ) é interessante em situações em que o destino das frutas seja um mercado mais exigente ou a exportação, pois produzem frutos maiores e melhor valorizados comercialmente. Entretanto, apresentam baixa produtividade em relação às densidades mais amplas, nos primeiros três ciclos. Comparativamente, a densidade de 1.333 plantas ha $^{-1}$ não apresentou vantagem em relação à densidade de 1.666 plantas ha ${ }^{-1}$, no que se refere à produtividade e ao tamanho do fruto. Além disto, é comum ocorrer, nos bananais menos adensados, perdas de plantas,

Tabela 8. Duração (meses) dos quatro primeiros ciclos de bananeiras 'Nanicão' submetidas a diferentes densidades e distribuições de plantio, em Piracicaba, $\mathrm{SP}^{(1)}$.

\begin{tabular}{|c|c|c|c|c|c|}
\hline \multirow{2}{*}{$\begin{array}{l}\text { Sistema de } \\
\text { espaçamento }\end{array}$} & \multicolumn{4}{|c|}{ Número de plantas ha ${ }^{-1}$} & \multirow[t]{2}{*}{ Média } \\
\hline & 3.333 & 2.222 & 1.666 & 1.333 & \\
\hline & \multicolumn{5}{|c|}{$1^{\circ}$ ciclo } \\
\hline Retângulo & 19,5 & 19,4 & 19,6 & 19,3 & $19,5 \mathrm{a}$ \\
\hline Triângulo & 19,3 & 19,9 & 19,4 & 19,5 & $19,5 \mathrm{a}$ \\
\hline \multirow[t]{2}{*}{ Média } & $19,4 \mathrm{~A}$ & $19,6 \mathrm{~A}$ & $19,5 \mathrm{~A}$ & $19,4 \mathrm{~A}$ & \\
\hline & \multicolumn{5}{|c|}{$2^{\underline{o}}$ ciclo } \\
\hline Retângulo & 10,6 & 9,5 & 8,9 & 7,6 & $9,2 \mathrm{a}$ \\
\hline Triângulo & 10,1 & 8,6 & 9,0 & 7,3 & $8,8 \mathrm{a}$ \\
\hline \multirow[t]{2}{*}{ Média } & $10,4 \mathrm{~A}$ & $9,0 \mathrm{~B}$ & $9,0 \mathrm{~B}$ & $7,5 \mathrm{C}$ & \\
\hline & \multicolumn{5}{|c|}{$3^{\circ}$ ciclo } \\
\hline Retângulo & 12,9 & 11,6 & 10,0 & 9,8 & $11,1 \mathrm{a}$ \\
\hline Triângulo & 12,3 & 11,6 & 10,5 & 10,2 & $11,2 \mathrm{a}$ \\
\hline \multirow[t]{2}{*}{ Média } & $12,6 \mathrm{~A}$ & $11,6 \mathrm{~A}$ & $10,3 \mathrm{~B}$ & $10,0 \mathrm{~B}$ & \\
\hline & \multicolumn{5}{|c|}{$4^{\circ}$ ciclo } \\
\hline Retângulo & 12,8 & 10,0 & 8,8 & 9,0 & $10,2 \mathrm{a}$ \\
\hline Triângulo & 12,7 & 9,9 & 9,0 & 8,8 & $10,1 \mathrm{a}$ \\
\hline Média & $12,7 \mathrm{~A}$ & $9,9 \mathrm{~B}$ & $8,9 \mathrm{~B}$ & $8,9 \mathrm{~B}$ & \\
\hline
\end{tabular}


por problemas de tombamento provocadas por ventos fortes, o que prejudica o estande da cultura e requer a operação de replantio.

A densidade média (2.222 plantas ha-1) constitui a melhor opção para a cultivar e região, pois apresenta produtividade superior à das baixas densidades, e produz frutos com tamanho semelhante. A tendência de manutenção da produtividade anual após o quarto ciclo também favorece a escolha desta densidade.

Entre os sistemas de espaçamento, poder-se-ia optar pelo sistema triângulo, pois as condições que oferece aumentam a massa do cacho, principalmente no primeiro ciclo, e promovem pequeno incremento na produtividade ao longo dos ciclos.

\section{Conclusões}

1. O aumento da densidade de plantio de bananeiras 'Nanicão', de 1.333 para 3.333 plantas ha- $^{-1}$, diminui a massa do cacho em 15 a $20 \%$.

2. A produtividade na maior densidade (3.333 plantas ha-1) é significativamente superior até o terceiro ciclo; no quarto ciclo, não há diferença quanto à produtividade entre densidades variando de 1.333 a 3.333 plantas ha-1 $\left(3,0\right.$ a 7,5 $\mathrm{m}^{2}$ planta $\left.^{-1}\right)$.

3. O tamanho e a massa do fruto são menores à medida que a densidade aumenta.

4. O sistema de espaçamento em triângulo fornece condições favoráveis à obtenção de maior massa do cacho no primeiro ciclo e pequeno aumento na produtividade em comparação com o sistema retângulo.

\section{Referências}

BELALCÁZAR CARVAJAL, S. L. El cultivo de plátano en el trópico. Cali : Feriva, 1991. 376 p.

CHATTOPADHYAY, P. K.; BHOWNIK, D. J.; MATI, S. C.; BOSE, T. K. Optimum planting density for plant and ratoon crops of 'Giant Governor' Cavendish banana in West Bengal. Indian Journal of Agricultural Sciences, New Delhi, v. 55, n. 1, p. 17-21, 1985.

CHUNDAWAT, B. S.; DAVA, S. K.; PATEL, N. L. Effect of close planting in the yield and quality of Lacatan banana. Indian Journal of Agricultural Sciences, New Delhi, v. 53 , n. 6 , p. $470-472,1983$.
DANIELLS, J. W.; O'FARREL, P. J.; CAMPBELL, S. J. The response of bananas to plant spacing in double rows in North Queensland. Queensland Journal of Agricultural and Animal Sciences, Brisbane, v. 42, n. 1, p. 45-51, 1985.

GOMES, J. A.; NÓBREGA, A. C.; ANDERSEN, O. Densidade de plantio da bananeira cultivar Prata (grupo AAB), na região produtora do Estado do Espírito Santo. In: CONGRESSO BRASILEIRO DE FRUTICULTURA, 7. , 1984, Florianópolis. Anais... Florianópolis : Sociedade Brasileira de Fruticultura/EMPASC, 1984. v. 1, p. 237-249.

ISRAELI, Y.; PLAUT, Z.; SCHWARTZ, A. Effect of shade on banana morphology, growth and production. Scientia Horticulturae, Amsterdam, v. 62, n. 1/2, p. 45-56, 1995.

JANICK, J. A ciência da horticultura. Rio de Janeiro : Freitas Bastos, 1968. 485 p.

KHOLI, R. R.; CHACKO, E. K.; RANDHAWA, G. S. Effect of spacing and nutrition on growth and fruit yield of 'Robusta' banana. Indian Journal of Agricultural Sciences, New Delhi, v. 46, n. 8, p. 382-385, 1976.

KLUGE, R. A. Densidade e sistema de espaçamento de bananeiras 'Nanicão' (Musa AAA subgrupo Cavendish). Piracicaba : ESALQ, 1999. 105 p. Tese de Doutorado.

LICHTEMBERG, L. A. Espaçamento e desbaste para bananeiras. Informativo da Sociedade Brasileira de Fruticultura, Cruz das Almas, v. 3, n. 3, p. 15-16, 1984.

LICHTEMBERG, L. A.; HINZ, R. H.; MALBURG, J. L. Espaçamento e desbaste para banana Nanicão em solo de encosta do litoral norte de Santa Catarina. Revista Brasileira de Fruticultura, Cruz das Almas, v. 12, n. 1, p. 53-61, 1990.

LICTHEMBERG, L. A.; HINZ, R. H.; MALBURG, J. L.; STUKER, H. Crescimento e duração dos cinco primeiros ciclos da bananeira Nanicão sob três densidades de plantio. Revista Brasileira de Fruticultura, Cruz das Almas, v. 19, n. 1, p. 15-23, 1997.

LICHTEMBERG, L. A.; HINZ, R. H.; MALBURG, J. L.; STUKER, H. Effect of three spacing on yield of 'Nanicão' banana in Southern Brazil. Acta Horticulturae, Leuven, n. 490, p. 181-186, 1998.

LICHTEMBERG, L. A.; MALBURG, J. L.; HINZ, R. H. Effect of planting density on yield and cycle duration of 'Nanicão' banana in Southern Brazil. Interamerican Society for Tropical Horticulture Proceedings, Homestead, v. 40, p. 232-235, 1996. 
LICHTEMBERG, L. A.; MALBURG, J. L.; HINZ, R. H. Espaçamento e desbaste para banana 'Enxerto'. In: CONGRESSO BRASILEIRO DE FRUTICULTURA, 9., 1987, Campinas. Anais... Campinas : Sociedade Brasileira de Fruticultura, 1988. v. 1, p. 161-169.

MATTOS, J. R.; SIMÃO, S.; CAMPOS, H. Influência do espaçamento no peso dos cachos da bananeira. O Solo, Piracicaba, v. 62, n. 2, p. 51-60, 1970.

MELIN, P.; PLAUD, G.; DU MANTCEL, H. T. Influence $\mathrm{du}$ mode de conduite de bananier plantain sur l'intensification de la culture. Fruits, Paris, v. 31, n. 11, p. 669-671, 1976.

OBIEFUNA, J. C.; MAJUMDER, P. K.; UCHEAGWU, A. C. Spacing and sucker management in the commercial plantain production in the rainforest belt of Nigeria. Annals of Applied Biology, Wellesbourne, v. 101, n. 2, p. 391-396, 1982.

PEREIRA, A. R. Competição intra-específica entre plantas cultivadas. O Agronômico, Campinas, v. 41, n. 1 , p. 5-11, 1989.

ROBINSON, J. C. Systems of cultivation and management. In: GOWEN, S. (Ed.). Bananas and plantain. London : Chapman \& Hall, 1995. p. 15-65.

ROBINSON, J. C.; NEL, D. J. The influence of banana (cv. Williams) plant density and canopy characteristics on ratoon cycle interval and yield. Acta Horticulturae, Leuven, n. 175, p. 227-232, 1986.

ROBINSON, J. C.; NEL, D. J. Plant density studies with banana (cv. Williams) in a subtropical climate. I. Vegetative morphology, phenology and plantation microclimate. Journal of Horticultural Science, Ashford, v. 63, n. 2, p. 303-313, 1988.
ROBINSON, J. C.; NEL, D. J. Plant density studies with banana (cv. Williams) in a subtropical climate. II. Components of yield and seasonal distribution of yield. Journal of Horticultural Science, Ashford, v. 64, n. 2, p. 211-222, 1989.

ROBINSON, J. C.; NEL, D. J.; BOWER, J. P. Plant density studies with banana (cv. Williams) in a subtropical climate. III. The influence of spatial arrangement. Journal of Horticultural Science, Ashford, v. 64, n. 4, p. 513-519, 1989.

SANTOS, P. J. Estudo do comportamento da bananeira (Musa acuminata, Colla), cv. Nanica em diferentes espaçamentos. Viçosa : UFV, 1977. 26 p. Dissertação de Mestrado.

SIMÃO, S. Tratado de fruticultura. Piracicaba : FEALQ, $1998.760 \mathrm{p}$.

SIMMONDS, N. W. Bananas. 2. ed. London : Longmans, 1982. 512 p. (Tropical Agriculture Series).

SOTO BALLESTERO, M. S.; SOTO, E.; SOLÍS, P.; LÓPEZ, A. Siembra y operaciones de cultivo. In: SOTO BALLESTERO, M. S. Bananos: cultivo y comercialización. San José : Litografia e Imprenta Lil, 1992. p. 211-265.

STOVER, R. H.; SIMMONDS, N. W. Bananas. 3. ed. New York : Longmans, 1987. 468 p. (Tropical Agriculture Series).

TURNER, D. W. Bananas and plantains. In: SCHAFFER, B.; ANDERSEN, P. C. (Ed.). Handbook of environmental physiology of fruit crops: sub-tropical and tropical crops. Boca Raton : CRC, 1994. v. 2, p. 37-64.

VIDAL-TORRADO, P.; SPAROVEK, G. Mapa pedológico detalhado do campus Luiz de Queiroz, Universidade de São Paulo. Piracicaba : ESALQ, 1993. Escala 1:10. 000 . 